\title{
BRST Field Theory of Relativistic Particles
}

\author{
J.W. van Holten* \\ NIKHEF-H, Amsterdam (NL)
}

August 31, 2018

\begin{abstract}
A generalization of BRST field theory is presented, based on wave operators for the fields constructed out of, but different from the BRST operator. We discuss their quantization, gauge fixing and the derivation of propagators. We show, that the generalized theories are relevant to relativistic particle theories in the Brink-Di Vecchia-Howe-Polyakov (BDHP) formulation, and argue that the same phenomenon holds in string theories. In particular it is shown, that the naive BRST formulation of the BDHP theory leads to trivial quantum field theories with vanishing correlation functions.
\end{abstract}

${ }^{*}$ Research supported by the Stichting F.O.M. 


\section{Introduction}

The dynamics of relativistic point particles in external electro-magnetic and gravitational fields is characterised by the mass-shell condition

$$
g^{\mu \nu}\left(p_{\mu}-q A_{\mu}\right)\left(p_{\nu}-q A_{\nu}\right)+m^{2} c^{2}=0,
$$

where $A_{\mu}$ is the electro-magnetic vector potential, and $g^{\mu \nu}$ the inverse space-time metric. For particles with spin, eq.(1) is modified [1]-[何, but this modification is not essential for this paper. Therefore we consider scalar particles only.

Although in classical point-particle mechanics eq.(1) is a consequence of the equations of motion, in field theory it is the starting point for defining the dynamics of the corresponding fields: the classical field equations are obtained by reinterpreting the quantities appearing in this equation as linear operators. These operators act on fields taking values in the some representation of the Lorentz group; for reasons mentioned above, in this paper we discuss scalar fields only. Finally, the fields themselves may be taken to represent the one-particle states of a corresponding relativistic quantum field theory.

It is well-known, that equations like (1) arise in classical mechanics as firstclass constraints in a theory with local reparametrization invariance [5]-[8]. Therefore the corresponding operator equations can be realized in terms of a BRST operator $\Omega$, which is nilpotent:

$$
\Omega^{2}=0,
$$

and the cohomology classes of which correspond to the solutions of the classical field equations::

$$
\{\Omega \Psi=0 \wedge \Psi \neq \Omega \Lambda\} \Leftrightarrow \mathcal{H} \Psi=0 .
$$

Here $\Psi$ is the classical field (or the corresponding one-particle quantum state vector), and $\mathcal{H}$ is the appropriate wave operator -in this case the Klein-Gordon operator, with proper inclusion of the coupling to electro-magnetic and gravitational background fields.

The definition of physical fields in terms of BRST cohomology, as in eq.(33), can be expressed naturally in terms of a classical variational principle. Define an action

$$
S_{0}=1 / 2(\Psi, \Omega \Psi)
$$

where $(\Phi, \Psi)$ denotes an inner product w.r.t. which $\Omega$ is self-adjoint ff; then $S_{0}$ is real and moreover invariant under BRST transformations:

\footnotetext{
${ }^{1}$ See [9, 10] and references therein

${ }^{2}$ Since $\Omega$ is nilpotent, this inner product necessarily has an indefinite signature $[13,15]$.
} 


$$
\Psi \rightarrow \Psi^{\prime}=\Psi+\Omega \Lambda
$$

with $\Lambda$ an arbitrary field taking values in the same space as $\Psi$. The invariance results from the nilpotency of the BRST operator, eq.(2). The variation of the action (14) vanishes precisely for those fields $\Psi$ which satisfy eqs.(3), and which are defined only modulo a transformation of type (5).

A quantum field theory, the one-particle states of which satisfy these BRSTtype of field equations, can now be constructed by defining a generating functional for Green's functions as

$$
Z[J]=\int[D \Psi] \exp i\left\{S_{0}-(J, \Psi)\right\} .
$$

Taking the integral naively as the integral over all classical configurations $\Psi$ would of course lead to a divergent expression, due to the BRST gauge invariance (5). Hence an appropriate gauge fixing procedure modifying the integration measure must be introduced to make the functional integral well-defined. Note that physical sources must themselves be BRST-invariant:

$$
\Omega J_{\text {phys }}=0 \text {. }
$$

Finally, BRST-invariant self-interaction terms for the fields $\Psi$ can be added to the action (4). Eq.(6) thus provides a starting point for a BRST-covariant quantum field theory. In particular, it can be used to set up BRST-covariant perturbation theory.

In this paper we discuss the BRST construction of point-particle field theories in quite some generality and show, that it contains several ambiguities which need to be resolved before a well-defined theory is obtained. The procedure described here was introduced originally in the construction of string field theories [17, 18, but its implementation in the field theory of point particles has received less attention?. The formal aspects of our results may be of some relevance in the context of string field theory as well.

\section{Gauge fixing}

In this section we take the BRST-operator $\Omega$, which specifies the field content and dynamics of the physical system, as given and discuss the implementation of the procedure to construct the generating functional for Green's functions of the quantum field theory, as sketched in the introduction.

First we note that the action (44) is not the most general BRST-invariant quadratic action one can construct given the inner product $(\Phi, \Psi)$. Rather, we can define a whole class of BRST-invariant actions specified by an additional operator $G$ with the property

\footnotetext{
${ }^{3}$ See however, refs. 19, 16]
} 


$$
G \Omega=(G \Omega)^{\dagger}=\Omega G^{\dagger}
$$

where a dagger denotes the adjoint w.r.t. the inner product introduced above. The action

$$
S_{G}=1 / 2(\Psi, G \Omega \Psi)
$$

is then both BRST-invariant and real. Of course, as long as $G$ is invertible, we can redefine the fields so as to bring the action back into the form (何). However, $G$ may also be singular (for example, if it is a projection operator) and then the expression (9) can not be rewritten in this form. A situation of this kind is actually encountered in the examples discussed below.

As mentioned, the BRST-invariance makes the naive functional integral

$$
Z_{G}[J]=\int[D \Psi] \exp i\left\{S_{G}-(J, \Psi)\right\}
$$

ill-defined. In order to construct a better-defined quantum theory one must restrict the integration to BRST-inequivalent configurations $\Psi$. A procedure for finding such gauge-fixed configurations was described in refs. [11-16. Briefly, the idea is to introduce a nilpotent co-BRST operator ${ }^{*} \Omega$ with

$$
\begin{aligned}
& \text { (i) } \quad * \Omega^{2}=0, \\
& \text { (ii) } \quad\left\{\Omega,{ }^{*} \Omega\right\}=\Delta,
\end{aligned}
$$

where we suppose the inverse $\Delta^{-1}$ to exist in the space of fields interacting with external sources. It also follows from the graded Jacobi identity that

$$
[\Delta, \Omega]=0, \quad\left[\Delta,{ }^{*} \Omega\right]=0 .
$$

The definition of * $\Omega$ is not unique; in fact one can generally find as many different co-BRST operators as there are different choices of gauge for the underlying reparametrization symmetry [15], [16].

Now we fix the BRST invariance by restricting the functional integral to field configurations such that

$$
G^{*} \Omega \Psi=0 .
$$

Reality of this condition requires the gauge-fixing operator to be hermitean:

$$
G^{*} \Omega={ }^{*} \Omega G^{\dagger}
$$

cf. eq.(8). The invertibility of $\Delta$ then implies that this gauge is well-defined (it intersects each BRST orbit once). Namely, the classical field equation obtained by varying the effective action in eq.(10) is 


$$
G \Omega \Psi=J
$$

which requires $J$ to be BRST-invariant for consistency. Now the restriction (13) together with eq.(14) implies

$$
{ }^{*} \Omega \Omega G^{\dagger} \Psi=\Delta G^{\dagger} \Psi={ }^{*} \Omega J
$$

and this equation has the solution

$$
G^{\dagger} \Psi=\Delta^{-1 *} \Omega J
$$

From this result we directly obtain the classical (i.e., tree-level) propagator in the singular gauge, the analogue of the Landau gauge in electro-dynamics. Again, we distinguish two cases: if $G$ has an inverse, we immediately have

$$
<\Psi \Psi>_{\text {tree }}=\frac{\delta \Psi}{\delta J}=\frac{1}{\Delta G^{\dagger}} * \Omega
$$

On the other hand, if $G$ is not invertible, consistency requires that we can write

$$
J=G K .
$$

In this case we obtain a tree-level propagator for the field $\Phi=G^{\dagger} \Psi$ as follows:

$$
<\Phi \Phi>_{\text {tree }}=\frac{\delta \Phi}{\delta K}=\frac{1}{\Delta} * \Omega G .
$$

In the functional integral the gauge condition can be implemented as usual by inserting a delta-functional $\delta\left[G^{*} \Omega \Psi\right]$ in the functional integral, accompanied by the corresponding Faddeev-Popov determinant. According to the standard FaddeevPopov-'t Hooft prescription including a Lagrange multiplier field $\Sigma$, this determinant can be written using Grassmann-odd ghost fields $(B, C)$ in the usual way, leading to an expression of the type

$$
\begin{aligned}
Z_{G}[J]= & \int[D \Psi][D \Sigma][D B][D C] \\
& \exp i\left\{S_{G}-(J, \Psi)-\frac{\alpha}{2}(\Sigma, T G \Sigma)-\left(\Sigma, T G^{*} \Omega \Psi\right)-\left(B, T G^{*} \Omega \Omega C\right)\right\} .
\end{aligned}
$$

where $\alpha T$ is a gauge-fixing operator to be chosen so as to make the full effective quantum action well-defined. Shifting the field $\Sigma$ to

$$
\Sigma^{\prime}=\Sigma+\frac{1}{\alpha} G^{*} \Omega \Psi,
$$

we can rewrite this in the form 


$$
\begin{aligned}
Z_{G}[J]= & \int[D \Psi]\left[D \Sigma^{\prime}\right][D B][D C] \\
& \exp i\left\{S_{G}+\frac{1}{2 \alpha}\left(\Psi,{ }^{*} \Omega T G^{*} \Omega \Psi\right)-\frac{\alpha}{2}\left(\Sigma^{\prime}, T G \Sigma^{\prime}\right)-\left(B, T G^{*} \Omega \Omega C\right)-(J, \Psi)\right\} .
\end{aligned}
$$

Unfortunately, this expression is again ill-defined, because now the ghost action is invariant under BRST-transformations of the ghosts $(B, C)$. Therefore one also needs to restrict the integration over the ghost fields in the same way, leading in the end to an infinite tower of ghost-for-ghosts with alternating Grassmann parity. However, at least if there are no additional self-interactions of the fields $\Psi$ we see that the functional integral over $\Psi$ and the ghosts $(B, C)$ factorizes. Now define the operator $E$ by

$$
G E=\left\{{ }^{*} \Omega, T\right\} G
$$

in the examples in this paper one can actually choose the gauge-fixing operator $T$ in such a way that $E=1$. Provided $G$ is invertible we can now write the integral over the $\Psi$ fields as

$$
Z_{G}[J]=Z_{G}[0] \exp -\frac{i}{2}\left(J, M^{-1} G^{-1} J\right)
$$

where

$$
M=\Omega+\frac{1}{\alpha} E^{*} \Omega
$$

and

$$
M^{-1}=\frac{1}{\Delta}\left({ }^{*} \Omega+\Omega \frac{\alpha}{E}\right) .
$$

Note, that in the limit $\alpha \rightarrow 0$ we reobtain the propagator (18), as anticipated. If $G$ is not invertible, we have to replace this expression by

$$
Z_{G}[K]=Z_{G}[0] \exp -\frac{i}{2}\left(K, G M^{-1} K\right) .
$$

If self-interactions of the $\Psi$ fields are added to the action, these expressions can still be used as the starting point for BRST-invariant perturbation theory in the standard fashion: replace the interactions of the $\Psi$ by vertex operators, obtained by substitution of the functional derivatives $\delta / \delta J$ for each $\Psi$ in the interaction term. In this context we remark, that in general the introduction of self-interactions modifies the BRST transformation rules so as to become $\Psi$ dependent. This also introduces interactions of $\Psi$ with the ghost fields $(B, C)$, which have to be treated similarly. 


\section{Point particles}

The classical Lagrangian for a scalar point particle with mass $m$ and charge $q$ moving in external fields $g_{\mu \nu}(x)$ and $A_{\mu}(x)$ is

$$
L=\frac{m}{2 e} g_{\mu \nu} \dot{x}^{\mu} \dot{x}^{\nu}+q A_{\mu} \dot{x}^{\mu}-\frac{1}{2} m e .
$$

Here $x^{\mu}$ are the position co-ordinates, a dot denotes the proper-time derivative and $e$ is the einbein variable which makes the theory reparametrization invariant on the world-line. We have also taken the velocity of light to be unity. In principle we could eliminate $e$ as a redundant degree of freedom in favor of a non-linear version of the theory, by replacing the einbein by the expression

$$
e=\sqrt{-g_{\mu \nu} \dot{x}^{\mu} \dot{x}^{\nu}}
$$

as suggested by its classical equation of motion. However, in order to keep the analogy with higher-dimensional reparametrization invariant theories -like Einstein gravity - in which the base-space metric has dynamical degrees of freedom as close as possible, we prefer to keep the einbein in the theory as an independent variable. In addition, in this formulation the Lagrangian remains a quadratic function of the velocities.

In the formulation (29), the theory has two first-class constraints originating in the local world-line reparametrization invariance:

$$
\mathcal{H}_{0}=0, \quad p_{e}=0 .
$$

Here $\mathcal{H}_{0}$ is $1 / e$ times the world-line Hamiltonian:

$$
2 m \mathcal{H}_{0}=g^{\mu \nu}\left(p_{\nu}-q A_{\mu}\right)\left(p_{\nu}-q A_{\nu}\right)+m^{2},
$$

which is identical with the constraint (11) upon taking $c=1$, and $p_{e}$ is the momentum conjugate to $e$.

In order to implement the BRST construction we introduce real Grassmannodd variables $\boldsymbol{g}=(b, c)$ with anti-real canonical momenta $\boldsymbol{\pi}=\left(\pi_{b}, \pi_{c}\right)$, having graded Poisson brackets

$$
\{\boldsymbol{g}, \boldsymbol{\pi}\}=i \mathbf{1} \text {. }
$$

Then we define the BRST-generator as

$$
\Omega=c \mathcal{H}_{0}+\pi_{b} p_{e} .
$$

The Poisson brackets of this quantity with the canonical co-ordinate and momentum variables generate BRST transformations in classical phase space, which 
leave $\mathcal{H}_{0}$ and the action as defined from the Lagrangian (29) invariant. Moreover, the graded Poisson bracket of $\Omega$ with itself vanishes:

$$
\{\Omega, \Omega\}=0,
$$

showing that these BRST transformations in classical phase space are nilpotent.

In field theory we replace the phase-space variables by operators with (anticommutators equal to $i$ times the corresponding Poisson brackets. In the coordinate picture this results in the substitutions

$$
\begin{array}{ll}
p_{\mu}=\frac{-i}{\sqrt[4]{-g}} \frac{\partial}{\partial x^{\mu}} \sqrt[4]{-g}, & p_{e}=-i \frac{\partial}{\partial e} \\
\pi_{b}=-\frac{\partial}{\partial b}, & \pi_{c}=-\frac{\partial}{\partial c}
\end{array}
$$

whilst $\left(x^{\mu}, e, b, c\right)$ denote multiplication by corresponding real (anti-)commuting c-numbers. Then we have

$$
\mathcal{H}_{0}=\frac{1}{2 m}\left(-D^{2}+m^{2}\right)
$$

where $D^{2}$ is the $U(1)$ and general-co-ordinate invariant laplacian on scalar functions, and

$$
\Omega=c \mathcal{H}_{0}+i \frac{\partial^{2}}{\partial b \partial e} .
$$

As the space of fields we take the polynomials in $(b, c)$ with co-efficients in the Cauchy completion of the set of square-integrable scalar functions of $\left(x^{\mu}, e\right)$. We define the scalar components by the expansion

$$
\Psi(b, c)=\psi-i b \psi_{b}+c \psi_{c}-i c b \psi_{c b}
$$

On this space we introduce an inner product

$$
(\Psi, \Phi)=i \int_{-\infty}^{\infty} d e \int_{-\infty}^{\infty} d^{n} x \int d b \int d c \sqrt{-g} \bar{\Psi} \Phi
$$

where $\bar{\Psi}$ is defined as

$$
\bar{\Psi}=\psi^{*}+i b \psi_{b}^{*}+c \psi_{c}^{*}-i c b \psi_{c b}^{*},
$$

the star denoting ordinary complex conjugation. With respect to this inner product both $\mathcal{H}_{0}$ and $\Omega$ are self-adjoint. In the following we call a field $\Psi$ real if it has real components $\psi_{i}$.

Noting, that 


$$
\Omega \Psi=\frac{\partial \psi_{b}}{\partial e}+c\left(\mathcal{H}_{0} \psi-\frac{\partial \psi_{c b}}{\partial e}\right)-i c b \mathcal{H}_{0} \psi_{b},
$$

it is now straightforward to show, that the non-trivial BRST cohomology classes consist of fields of the form

$$
\Psi(b, c)=-i b \psi_{b},
$$

which satisfy

$$
\left(-D^{2}+m^{2}\right) \psi_{b}=0, \quad \frac{\partial \psi_{b}}{\partial e}=0 .
$$

The first equation is the standard field equation for the wave function of a single scalar particle in external fields, whilst the second equation expresses the reparametrization invariance of the physical states of the theory: any worldline reparametrization (a redefinition of proper time) can be absorbed in a redefinition of $e$; since physical states $\Psi$ do not depend on $e$, they are ipso facto invariant under these reparametrizations.

Finally it follows from eq.(42) that the BRST transformations of the field components are:

$$
\begin{array}{rlrl}
\delta \psi & =\frac{\partial \xi_{b}}{\partial e}, & \delta \psi_{b} & =0 \\
\delta \psi_{c}=\mathcal{H}_{0} \xi-\frac{\partial \xi_{c b}}{\partial e}, & \delta \psi_{c b} & =\mathcal{H}_{0} \xi_{b} .
\end{array}
$$

Hence the $\psi_{b}$-component of the field is the only one inert under BRST transformations.

\section{Quantum theory of the scalar field}

Finally we turn to the construction of a quantum theory for the scalar field. Without undue loss of generality we may take this field to be real (in the sense explained above). As a first step we look for operators $G$ with the property (8). They are of the form

$$
G=\boldsymbol{n}+i c \boldsymbol{n}_{\boldsymbol{c}}+i \boldsymbol{n}_{\boldsymbol{b}} \frac{\partial}{\partial b}+i c \boldsymbol{n}_{\boldsymbol{c b}} \frac{\partial}{\partial b},
$$

where $\left(\boldsymbol{n}, \boldsymbol{n}_{\boldsymbol{b}}\right)$ are taken to commute with $\mathcal{H}_{0}$, and $\left(\boldsymbol{n}, \boldsymbol{n}_{\boldsymbol{c}}\right)$ with $p_{e}$. The term with $\boldsymbol{n}_{\boldsymbol{c} \boldsymbol{b}}$ is immaterial, because it vanishes upon multiplication with $\Omega$. Hence we may choose $\boldsymbol{n}_{\boldsymbol{c} \boldsymbol{b}}=0$ from the start. Furthermore we observe, that $G$ is invertible if and only if $\boldsymbol{n}^{-1}$ exists. 
As in eq.(9) we now obtain an action leading to the equations of motion (44) by taking

$$
S_{G}=1 / 2(\Psi, G \Omega \Psi),
$$

with the inner product as defined in eq.(40). In terms of components this gives:

$$
S_{G}=\int_{-\infty}^{\infty} d e \int_{-\infty}^{\infty} d^{n} x \sqrt{-g}\left\{\psi \boldsymbol{n} \mathcal{H}_{0} \psi_{b}+\psi_{c b} \boldsymbol{n} \frac{\partial \psi_{b}}{\partial e}-\frac{1}{2} \psi_{b}\left(\boldsymbol{n}_{\boldsymbol{b}} \mathcal{H}_{0}-i \boldsymbol{n}_{\boldsymbol{c}} \frac{\partial}{\partial e}\right) \psi_{b}\right\} .
$$

We observe, that the component $\psi_{c}$ does not occur in this expression. Indeed, such a component can always be gauged away by a BRST transformation leaving the action invariant.

As a result, if we define a BRST-invariant functional integral of the type eq. (10) the integration over this component is not damped by exponential factors, and hence will give rise to a divergent expression, as anticipated above. The solution to this problem has already been discussed, see eqs.(11-21). The procedure outlined in sect. 2 is suitable if we can find an appropriate co-BRST operator. Such a co-BRST operator is for example

$$
\begin{aligned}
* \Omega= & -\pi_{c} \\
& \mapsto \frac{\partial}{\partial c} .
\end{aligned}
$$

The corresponding BRST laplacian is then

$$
\Delta=\mathcal{H}_{0},
$$

which up to a factor $1 / 2 m$ is just the Klein-Gordon operator.

In order to complete the construction of the effective quantum action (23), we also specify an operator $T$ by

$$
T=c \text {. }
$$

As a consequence,

$$
E=1
$$

With this choice the gauge-fixed kinetic operator in the effective quantum action (23) becomes

$$
M=\Omega+\frac{1}{\alpha} * \Omega=c \mathcal{H}_{0}+i \frac{\partial^{2}}{\partial e \partial b}+\frac{1}{\alpha} \frac{\partial}{\partial c} .
$$

It follows that the gauge-fixed form of the propagator is 


$$
M^{-1}=\frac{2 m}{\left(-D^{2}+m^{2}\right)}\left(\frac{\partial}{\partial c}+i \alpha \frac{\partial^{2}}{\partial e \partial b}\right)+\alpha c .
$$

The gauge fixing terms in the action read in components

$$
\begin{aligned}
& \frac{1}{2 \alpha}\left(\Psi, G^{*} \Omega \Psi\right)= \\
& \quad \frac{1}{2 \alpha} \int_{-\infty}^{\infty} d e \int_{-\infty}^{\infty} d^{n} x \sqrt{-g}\left\{i \boldsymbol{n}_{c} \psi_{c b}+i \psi_{b} \boldsymbol{n}_{c} \psi_{c}+\psi_{c} \boldsymbol{n} \psi_{c b}+\psi_{c b} \boldsymbol{n} \psi_{c}+\psi_{c b} \boldsymbol{n}_{\boldsymbol{b}} \psi_{c b}\right\}
\end{aligned}
$$

Adding this term to $S_{G}$, eq.(48), gives the full expression for the scalar-field dependent terms in the effective action.

From the component results (48,55) it is easy to see that different choices of $G$ lead to physically quite different results. As a first example, consider the case $G=1$, or

$$
\boldsymbol{n}=1, \quad \boldsymbol{n}_{\boldsymbol{b}}=\boldsymbol{n}_{\boldsymbol{c}}=0 .
$$

Then the effective quantum action is

$$
S_{e f f}=\int_{-\infty}^{\infty} d e \int_{-\infty}^{\infty} d^{n} x \sqrt{-g}\left(\psi \mathcal{H}_{0} \psi_{b}+\psi_{c b}\left(\frac{\partial \psi_{b}}{\partial e}+\frac{1}{\alpha} \psi_{c}\right)+\text { ghost terms }\right)
$$

It follows, that for $\alpha \neq 0$ the functional integral for fields coupled to physical (BRST-invariant) sources becomes, after integrating out $\left(\psi, \psi_{c}, \psi_{c b}\right)$ :

$$
Z[j]=\mathcal{N} \int\left[D \psi_{b}\right] \delta\left[\mathcal{H}_{0} \psi_{b}\right] \exp -i \int_{-\infty}^{\infty} d e \int_{-\infty}^{\infty} d^{n} x \sqrt{-g} j \psi_{b},
$$

where $\mathcal{N}$ is a $\psi$-independent (but background field dependent) normalization factor coming from the integral over the ghosts. Therefore only classical configurations satisfying the Klein-Gordon equation contribute to the functional integral, and quantum fluctuations are suppressed. In particular, in this theory the 2-point function vanishes. One way to see this is to observe, that writing the $\delta$-functional as a Fourier integral the source term can be absorbed by a shift of the multiplier field. Hence the right-hand side is really independent of the source $j$. Clearly, this theory is not equivalent with the standard quantum theory of the scalar field.

In contrast, the standard scalar field theory is obtained for the singular choice

$$
\begin{aligned}
G & =c \frac{\partial}{\partial e}+2 i m \frac{\partial}{\partial b} \\
& =-G^{\dagger}
\end{aligned}
$$


which implies

$$
\boldsymbol{n}=0, \quad \boldsymbol{n}_{\boldsymbol{b}}=2 m, \quad \boldsymbol{n}_{\boldsymbol{c}}=p_{e}=-i \frac{\partial}{\partial e} .
$$

With this choice one finds for the effective quantum action

$$
\begin{aligned}
S_{\text {eff }}= & \int_{-\infty}^{\infty} d e \int_{-\infty}^{\infty} d^{n} x \sqrt{-g}\left(-\frac{1}{2} \psi_{b}\left(2 m \mathcal{H}_{0}-\frac{\partial^{2}}{\partial e^{2}}\right) \psi_{b}-\frac{1}{2 \alpha} \psi_{c} \frac{\partial \psi_{b}}{\partial e}\right. \\
& \left.-\frac{1}{16 \alpha m}\left(\frac{\partial \psi}{\partial e}\right)^{2}+\frac{m}{\alpha}\left(\psi_{c b}-\frac{1}{4 m} \frac{\partial \psi}{\partial e}\right)^{2}+\text { ghost terms }\right) .
\end{aligned}
$$

Integration over the $\psi_{c b}, \psi$ and $\psi_{c}$ now gives

$$
\begin{aligned}
Z[j] & =\mathcal{N}^{\prime} \int\left[D \psi_{b}\right] \delta\left[\frac{\partial \psi_{b}}{\partial e}\right] \exp i \beta \int_{-\infty}^{\infty} d e \int_{-\infty}^{\infty} d^{n} x \sqrt{-g}\left\{-\frac{1}{2} \psi_{b}\left(-D^{2}+m^{2}\right) \psi_{b}^{2}-j \psi_{b}\right\} \\
& =\frac{\mathcal{N}^{\prime}}{\sqrt{\operatorname{det}\left(-D^{2}+m^{2}\right)}} \exp \frac{i}{2} \int_{-\infty}^{\infty} d^{n} x \sqrt{-g} j \Delta_{K G}^{-1} j,
\end{aligned}
$$

where $\Delta_{K G}^{-1}$ denotes the Feynman propagator for the Klein-Gordon scalar field, and a factor $\beta$ has been introduced to normalize the integration over $e$. In view of the $\delta$-function contraint on $\psi_{b}$, it is legitimate to take the source to be $e$-independent from the start. We observe that although this theory does give the correct Green's functions for the scalar field, full equivalence with the standard scalar field theory is achieved only if the normalization factor $\mathcal{N}^{\prime}$, which results from integration over the ghosts, is independent of the background fields $\left(A_{\mu}, g_{\mu \nu}\right)$. This poses a strict constraint on possible ways to deal with the infinite tower of ghost-for-ghosts in the present formulation of the theory.

Note that both choices of $G$ discussed above yield the correct classical equations of motion. Nevertheless they correspond to different quantum theories, because they lead to different Green's functions for the fields. In order to investigate this point in some more detail, consider the class of interpolating gauges

$$
G=\lambda+\mu c \frac{\partial}{\partial e}+2 i m \nu \frac{\partial}{\partial b},
$$

where $(\lambda, \mu, \nu)$ are numerical parameters. For $\lambda=1$ and $\mu=\nu=0$ we reobtain the first case $G=1$, whilst for $\lambda=0$ and $\mu=\nu=1$ we get back the standard theory defined by the $G$ of eq.(59). Inserting this into eqs.(48,55) we first note, that for $\nu=0$ and $\lambda \neq 0$ we are always restricted to terms linear in the unphysical

\footnotetext{
${ }^{4}$ Effectively this represents a renormalization of $\hbar$.
} 
field components $\left(\psi, \psi_{c}, \psi_{c b}\right)$, which act as Lagrange multipliers in the functional integral and always lead to a $\delta$-function constraint requiring $\mathcal{H}_{0} \psi_{b}=0$ in the measure of the functional integral, like in eq.(58). Therefore we assume $\nu \neq 0$.

Now we know already that in this case $\lambda=0$ leads to the correct standard functional integral, eqs.(61,621). Therefore we also take $\lambda \neq 0$. Then after performing the shift of fields

$$
\begin{aligned}
& \psi_{c b}^{\prime}=\psi_{c b}+\frac{\lambda}{2 m \nu} \psi_{c}-\frac{\mu}{4 m \nu} \frac{\partial \psi}{\partial e}+\frac{\alpha \lambda}{2 m \nu} \frac{\partial \psi_{b}}{\partial e}, \\
& \psi_{c}^{\prime}=\psi_{c}-\frac{\mu}{2 \lambda} \frac{\partial \psi}{\partial e}+\left(\alpha+\frac{m \nu \mu}{\lambda^{2}}\right) \frac{\partial \psi_{b}}{\partial e},
\end{aligned}
$$

the effective action reads in components:

$$
\begin{aligned}
S_{\text {eff }}=\int_{-\infty}^{\infty} d e \int_{-\infty}^{\infty} d^{n} x \sqrt{-g} & \left(\frac{m \nu}{\alpha} \psi_{c b}^{\prime 2}-\frac{\lambda^{2}}{4 \alpha m \nu} \psi_{c}^{\prime 2}-\frac{\nu}{2} \psi_{b} \mathcal{H}_{e f f} \psi_{b}\right. \\
& \left.+\frac{\lambda}{2 m} \psi \mathcal{H}_{\text {eff }} \psi_{b}+\text { ghost terms }\right) .
\end{aligned}
$$

Here the effective wave operator is given by

$$
\mathcal{H}_{\text {eff }}=-D^{2}+m^{2}+\frac{m \mu^{2}}{2 \alpha \lambda^{2}} \frac{\partial^{2}}{\partial e^{2}} .
$$

The integration over the auxiliary field components $\left(\psi_{c}^{\prime}, \psi_{c b}^{\prime}\right)$ is trivial, but the intergral over $\psi$ is now seen to lead always to a $\delta$-function constraint $\mathcal{H}_{\text {eff }} \psi_{b}=0$ in the functional integral. We conclude, that with the exception of the singular case $\lambda=0, \nu \neq 0$ we always get trivial quantum field theories.

\section{Self-Interactions}

The above actions can without much difficulty be extended to include self-interactions of the scalar fields $\Psi$ [16]. The basic observation is, that since the $\psi_{b}$ component is inert under BRST transformations, any interaction involving only this component is automatically BRST-invariant under the original set of BRST transformations. Note that this situation differs from the case of string theories, in which both the action and the BRST transformation rules have to be modified.

Restricting ourselves to the case of polynomial interactions, we find that there are many different ways to write such interactions in terms of the BRST superfields (39). For the case of $G$ as in eq.(59), which is the only one of physical interest, a convenient choice is 


$$
\begin{aligned}
\Delta S_{\text {int }} & =\frac{-g_{n}}{n !} \int_{-\infty}^{\infty} d e \int_{-\infty}^{\infty} d^{n} x \int d b \int d c c b \sqrt{-g}\left(\frac{G \Psi}{2 m}\right)^{n} \\
& =\frac{-g_{n}}{n !} \int_{-\infty}^{\infty} d e \int_{-\infty}^{\infty} d^{n} x \sqrt{-g} \psi_{b}^{n} .
\end{aligned}
$$

Note, that in general the interacting theory does not take the form of a ChernsSimons like theory, as is the case of the open string. In particular, in the action (61) there are no gauge independent quadratic auxiliary field terms which could be used to cast the polynomial interactions for, say, the case $n=4$ into cubic form.

Finally we remark that since in the interacting scalar theory the BRST transformation rules are not modified, neither are the gauge-fixing and ghost terms in the effective quantum action (61).

\section{The ghost terms}

For the particular case of the theory defined by $G$ of eq.(59), and with the choice (49) for ${ }^{*} \Omega$ and (51) for $T$, the ghost terms in the effective action become particularly simple and can be seen to result in a background-independent factor in front of the functional integral, which may subsequently be ignored.

Indeed, substituting the explicit expressions into eq.(23) and denoting the components of $(\Sigma, B, C)$ by $\left(\sigma_{i}, \beta_{i}, \gamma_{i}\right)$, one obtains

$$
-\frac{\alpha}{2}\left(\Sigma^{\prime}, T G \Sigma^{\prime}\right)-\left(B, T G^{*} \Omega \Omega C\right)=\int_{-\infty}^{\infty} d e \int_{-\infty}^{\infty} d^{n} x \sqrt{-g}\left(-\alpha m \sigma_{b}^{2}+2 m \beta_{b} \gamma_{b}\right) .
$$

Even though the functional integral over the other ghost-field components is not damped, the result of further gauge fixing and the introduction of ghost-for-ghosts will only result in an over-all factor which is background-field independent and cancels from any physical Green's function in the theory. In fact, this factor is BRST invariant by itself and may be divided out from the start without spoiling BRST invariance of the theory. 


\section{Discussion}

As illustrated by the example of the scalar point particle, the BRST formulation of a quantum field theory is not always obtained from the naive action $(\Psi, \Omega \Psi)+$ interactions, but rather one needs the more general form (9). In the case of the scalar particle the effective kinetic operator is

$$
\Omega_{G} \equiv G \Omega=i c\left(D^{2}+\frac{\partial^{2}}{\partial e^{2}}-m^{2}\right) \frac{\partial}{\partial b} .
$$

The operator $\Omega_{G}$ is a Grassmann-even nilpotent operator. Obviously, transformations of the form $\delta \Psi=\Omega_{G} \Lambda$ leave the action invariant and the physical states belong to the cohomology classes of $\Omega_{G}$. However, the set of transformations generated by $\Omega_{G}$ is smaller than the BRST transformations generated by $\Omega$ itself, which we already know to be a full symmetry of the action $S_{G}$. Hence the cohomology of $\Omega_{G}$ cannot by itself completely determine the physical states. Indeed, the states obtained from the cohomology of $\Omega_{G}$ are zero modes of the effective wave operator

$$
\mathcal{H}_{\text {eff }}=-D^{2}-\frac{\partial^{2}}{\partial e^{2}}+m^{2},
$$

but they are not necessarily annihilated by the einbein momentum operator $p_{e}=$ $-i \partial / \partial e$. Hence this really describes a particle in a 5 -dimensional space-time, with $e$ acting as the fifth co-ordinate.

We may contrast this situation with the case in which we start from the Nambu-Goto type theory [0] in which the einbein $e$ is not an independent variable, but rather given by the non-linear expression (30). In that case, there is only one independent first-class constraint, the mass-shell condition $2 m \mathcal{H}_{0}=(p-q A)^{2}+$ $m^{2}=0$. The corresponding BRST operator is now the 'irreducible part' of $\Omega$ :

$$
\Omega_{\text {irr }}=c \mathcal{H}_{0} .
$$

Since there is no gauge variable $e$ present, which has to be eliminated from the physical states, we donot need to introduce more ghosts and we find that the physical states are defined directly by the cohomology of $\Omega_{i r r}$ in the space of polynomials $\Phi[c]$ in the ghost $c$ only. In this space the BRST-invariant action for the corresponding quantum field theory is simply

$$
\begin{aligned}
S & =m \int_{-\infty}^{\infty} d^{n} x \int d c \sqrt{-g} \Phi \Omega_{i r r} \Phi \\
& =1 / 2 \int_{-\infty}^{\infty} d^{n} x \sqrt{-g} \phi\left(-D^{2}+m^{2}\right) \phi,
\end{aligned}
$$

where $\phi$ is the lowest component of $\Phi$, which is invariant under the irreducible BRST transformations generated by $\Omega_{i r r}$. 
Actually the same result is known to hold in the case of the open bosonic string: the action of the type (72) is known to give the correct string field equations provided the BRST operator $\Omega$ is constructed from the dynamical Virasoro constraints only, as follows naturally starting from the Nambu-Goto action. Starting from the Brink-Di Vecchia-Howe-Polyakov action [20]-[22] with the 2dimensional metric as independent gauge degrees of freedom, one gets additional first-class constraints eliminating these metric degrees of freedom from physical states. Then the BRST operator contains extra terms constructed with the additional ghosts, and the action principle needs modifications of the type we have discussed here.

\section{References}

[1] B.S. Skagerstam and A. Stern, Phys. Scr. 24 (1981), 493

[2] J.W. van Holten, Proc. Sem. Math. Structures in Field Theories (1986-1987), CWI Syllabus vol. 26 (1990); eds. E.A. de Kerf and H.G.J. Pijls

[3] J.W. van Holten, Nucl. Phys. B356 (1991), 3

[4] I.B. Khriplovich, Novosibirsk preprint INR-89-1

[5] R. Casalbuoni, J. Gomis and G. Longhi, Nuov. Cim. 24A (1974), 249

[6] F.A. Berezin and M.S. Marinov, Ann. Phys. 104 (1977), 336

[7] L. Brink, P. Di Vecchia and P. Howe, Nucl. Phys. B118 (1977), 76

[8] A. Barducci, R. Casalbuoni and L. Lusanna, Nuov. Cim. 35A (1976), 377

[9] T. Kugo and I. Ojima, Suppl. Prog. Theor. Phys. 66 (1979), 1

[10] M. Henneaux, Phys. Rep. 126 (1985), 1

[11] J. Gervais, Nucl. Phys. B276 (1986), 339

[12] I. Bars and S. Yankielowicz, Phys. Rev. D35 (1987), 3878

[13] M. Spiegelglas, Nucl. Phys. B283 (1987), 205

[14] J.W. van Holten, Phys. Rev. Lett. 64 (1990), 2863; Nucl. Phys. B339 (1990), 158

[15] W. Kalau and J.W. van Holten, Nucl. Phys. B361 (1991), 233 
[16] H. Hüffel, Phys. Lett. B241 (1990), 369; Int. J. Mod. Phys. A6 (1991), 4985

[17] W. Siegel, Phys. Lett. 151B (1985), 391 and 396

[18] E. Witten, Nucl. Phys. B 268 (1986), 79; Nucl. Phys. B276 (1986), 291

[19] W. Siegel, Introduction to String Field Theory (World Scientific, Singapore, 1988)

[20] L. Brink, P. Di Vecchia and P. Howe, Phys. Lett. B65 (1976), 471

[21] S. Deser and B. Zumino, Phys. Lett. B65 (1976), 369

[22] A.M. Polyakov, Phys. Lett. B103 (1981), 207 